\title{
Reduction cranioplasty for macrocephaly with long-standing hydrocephalus and non-fused fontanelle in Chiari malformation type I
}

\author{
Jong-Il Choi • Eun Sang Dhong • Dong-Jun Lim • \\ Sang-Dae Kim
}

Received: 15 January 2014 / Accepted: 23 May 2014 /Published online: 6 June 2014

(C) Springer-Verlag Berlin Heidelberg 2014

\begin{abstract}
Introduction Because hydrocephalus is diagnosed and treated at an early stage in pediatric patients, pediatric neurosurgeons rarely encounter patients with hydrocephalic macrocephaly. There are even fewer cases of infants with long-standing hydrocephalus in whom macrocephaly progresses and is accompanied by skull defect due to malunion of suture lines despite long-term CSF diversion treatment.

Case report We report the case of a male infant with Chiari malformation type I who presented with congenital hydrocephalus and occipital encephalocele that progressed to hydrocephalic macrocephaly with frontal skull defect, despite numerous cerebrospinal fluid diversion operations. The patient eventually recovered successfully after reduction cranioplasty.
\end{abstract}

Keywords Chiari I malformation $\cdot$ Hydrocephalic macrocephaly · Bony defect · Reduction cranioplasty · Occipital encephalocele

\section{Introduction}

Fontanelle bulging and macrocephaly with bony defect due to persistent hydrocephalus despite adequate choice and timing of treatment options is extremely rare. Macrocephaly refers to a condition where the circumference of the head is greater than two standard deviations above the mean circumference for a

J.-I. Choi • D.-J. Lim · S.-D. Kim $(\bowtie)$

Department of Neurosurgery, Korea University Ansan Hospital,

Ansan, Korea

e-mail: neuron19@korea.ac.kr

E. S. Dhong

Department of Plastic surgery, Korea University Guro Hospital, Seoul, Korea given age. A massive head size may lead to difficulties with head positioning and movement control in a child. If this condition persists, complications like developmental disruption, kyphosis, or scoliosis may occur. When the cranium remains unclosed, facial deformities, vulnerability to trauma, and cosmetic issues may arise. In addition, a massive head size that results in significant deformity may lead to psychosocial problems [1]. Therefore, macrocephaly with skull defect needs to be corrected at an appropriate time.

Macrocephaly most commonly results from untreated or inadequately treated hydrocephalus [1-3] or from expanding chronic subdural hematomas [4, 5]. Various kinds of congenital anomalies may also be responsible for postnatal hydrocephalus. The treatment options and prognosis for an infant with Chiari I malformation secondary to hydrocephalus from an occipital encephalocele are less well known due to its low prevalence.

The treatment of hydrocephalic macrocephaly with frontal skull defect is an uncommon circumstance, rarely faced by pediatric neurosurgeons. There are few reported cases of longstanding hydrocephalus in Chiari malformation type III that progress to macrocephaly with skull defect due to suture malunion despite various cerebrospinal fluid (CSF) diversion treatments. Therefore, we herein describe a case of hydrocephalic macrocephaly with bony defect in Chiari malformation type I, and its treatment with reduction cranioplasty, which markedly improved the quality of life of the patient and achieved good cosmetic results.

\section{Case report}

A male baby with low body weight $(2,530 \mathrm{~g})$ was born at full term by cesarean section, while his twin brother was born with normal birth weight and no specific complications. An antenatal ultrasound investigation had already revealed an 
occipital encephalocele in the affected child. After birth, this was clinically confirmed, as the newborn presented with an encephalocele that measured $2 \times 2.5 \mathrm{~cm}$ in size and was entirely covered with meninges.

Magnetic resonance imaging (MRI) was performed the day after delivery and showed severe hydrocephalus and an occipital encephalocele. The cerebral aqueduct and fourth ventricle were collapsed, and consequent dilatation of the lateral and third ventricles were noted, along with the herniation of the lower brainstem and cerebellum into the cervical defect (Fig. 1).

In order to prevent infection or rupture of the encephalocele sack, surgical closure of the defect was performed and was successful. Postoperatively, the hydrocephalus worsened, so a ventriculo-peritoneal (V-P) shunt was placed. On physical examination after the V-P shunt operation, the patient's head circumference continued to increase gradually with massive frontal bossing, and the function of the shunt was not satisfactory. On the follow-up MRI scan, dilatation of the lateral and third ventricles, slight dilatation of the fourth ventricle, and evidence of tonsillar descent below the rim of the foramen magnum were noted (Fig. 2).

Foramen magnum decompression and endoscopic third ventriculostomy (ETV) were performed to correct the associated obstructive hydrocephalus. However, ventriculomegaly persisted even after the ETV, and shunt infection had developed. The shunt device was removed. Extra-ventricular drainage (EVD) and antibiotic therapy took place multiple times, and a shunt reoperation was performed.

A follow-up brain CT scan was done after the shunt reoperation, and there was an improvement in the long-standing hydrocephalus. However, macrocephaly was still evident and the anterior fontanelle still bulged open 32 months postnatally.

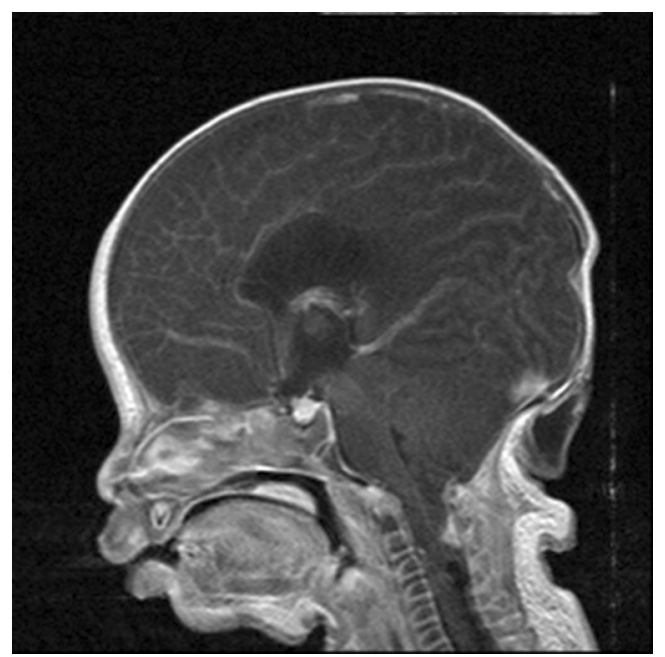

Fig. 1 Preoperative MRI scan (T1-weighted mid-sagittal scan) clearly demonstrating an occipito-cervical meningoencephalocele, sized approximately $2 \times 2.5 \mathrm{~cm}$ with hypoplastic cerebellar structures jutting into the malformed sac. Cerebellar tonsillar herniation below the fourth ventricle, together with collapsed cerebral aqueduct and fourth ventricle are seen

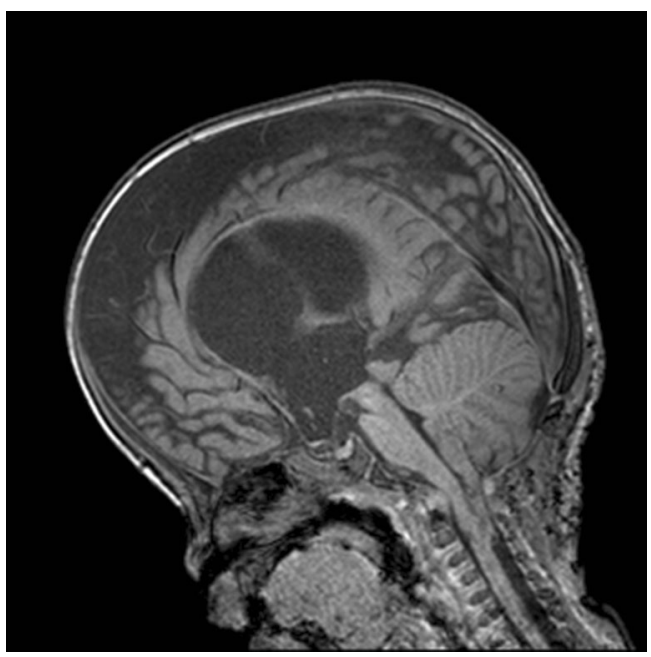

Fig. 2 Preoperative MRI scan (T1-weighted mid-sagittal scan) demonstrating tonsillar herniation in the upper cervical level. Dilated lateral, third ventricle, and slightly dilated fourth ventricle are seen

The defect measured $57 \times 61 \mathrm{~mm}$ in size along the anterior fontanelle. The skull was boat shaped; the anterior-posterior diameter was $168 \mathrm{~mm}$ and the bi-temporal width measured $120 \mathrm{~mm}$. The cranial index was 0.71 (Fig. 4a).

The patient underwent modified pi-procedure of reduction cranioplasty for cranial vault reshaping (Fig. 3). The coronal bone flap was advanced to the midline to cover the bone defect and two paired sagittal-parietal bone flaps were removed and grafted at the anterior fontanelle.

Multiple barrel stave osteotomy at the level of the squamosal suture was performed, allowing the brain to expand laterally on both sides during intraoperative skull shortening. Postoperatively, the non-fused fontanelle with skull defect was occluded. The operative time (defined as the beginning of anesthesia until the end of anesthesia) was $6.5 \mathrm{~h}$, and the blood loss associated with the surgery was $300 \mathrm{ml}$.

After the successful correction of macrocephaly, the skull vault circumference decreased from 58 to $53 \mathrm{~cm}$. The cranial index had changed to 0.75 (Fig. 4b). There were no postoperative complications.

Early in infancy, the patient showed substantial developmental delays, manifested by failure to meet routine

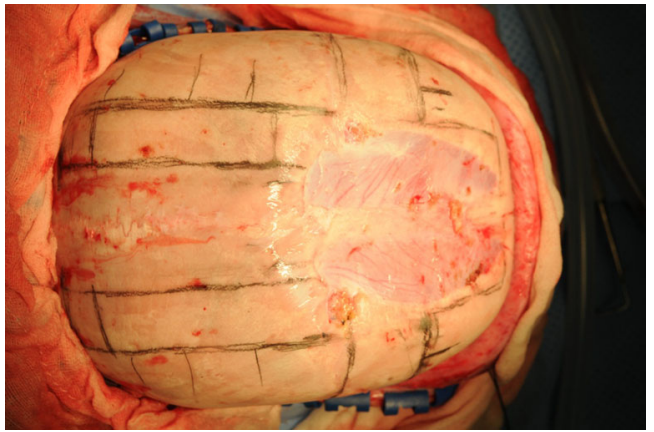

Fig. 3 Intraoperative view demonstrating macrocephaly with bony defect due to non-fused fontanelle 
Fig. 4 Pre- (a) and postoperative (b) 3D skull CT scans after successful correction of macrocephaly and occlusion of frontal bony defect with the described technique
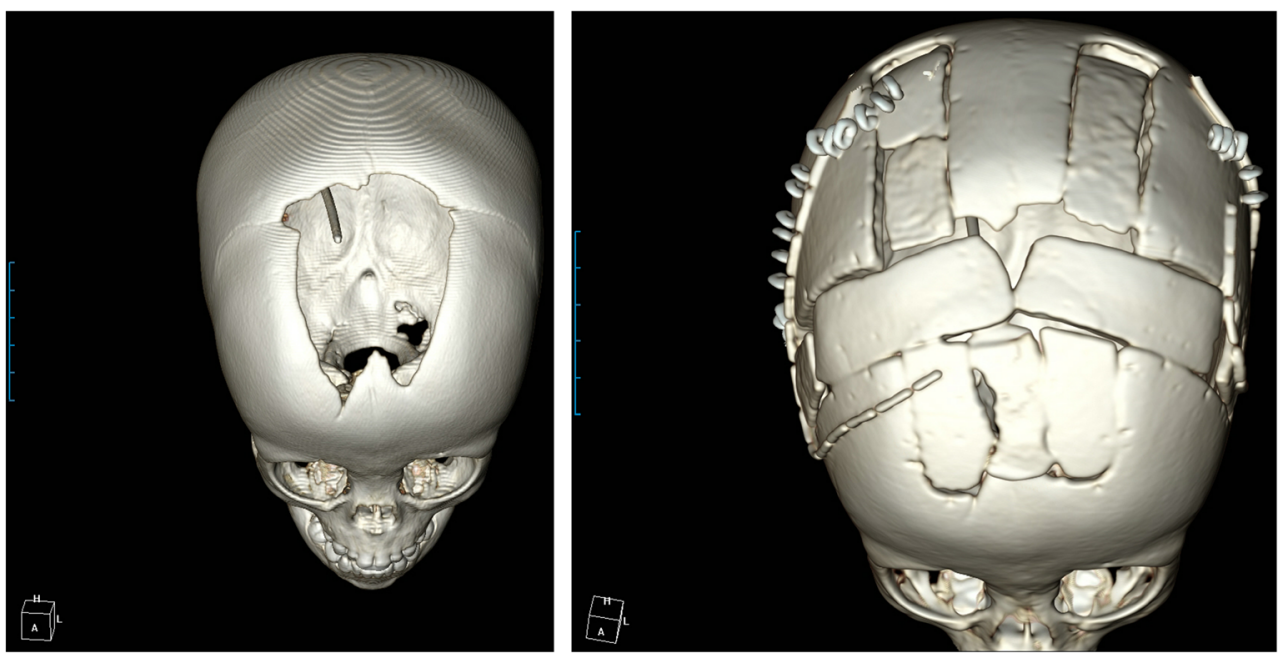

developmental milestones. However, slow neurological improvement was observed during the overall course of development.

\section{Discussion}

The Chiari malformations are a complex of hindbrain deformities associated with spinal abnormalities and hydrocephalus. The most frequent varieties are types I and II, the latter being almost invariably associated with meningomyelocele. Type III is the rarest of the Chiari malformations and is characterized anatomically by its association with a high cervical or occipital encephalocele, and many of the abnormalities usually found in the second type, such as a small posterior cranial fossa, caudal displacement of cerebellar tonsils and vermis, medullary kinking, tectal beaking, and obvious hydrocephalus [6-8]. Our case is a rare example of Chiari I malformation secondary to hydrocephalus from an occipital encephalocele.

Newborns with Chiari malformation frequently suffer from various disorders, such as respiratory insufficiency, dysphagia with secondary aspiration, dysfunction of the cranial nerves, spastic or decreased muscle tone, and loss of bladder or sphincter function. In addition, hydrocephalus affects only a minority of patients with Chiari type I malformation [9] but is closely related to the previously mentioned disorders. Therefore, it is important to manage hydrocephalus during the initial stages.

Our patient had been successfully treated with surgery for his occipito-cervical encephalocele on his first day of life. However, various methods of CSF diversion normally used in congenital hydrocephalus, including V-P shunt, foramen magnum decompression, and endoscopic third ventriculostomy (ETV), all failed to correct the hydrocephalus, and eventually, the patient developed shunt malfunction and infection that led to persistent hydrocephalus. Although this long-standing hydrocephalus was then corrected with a V$\mathrm{P}$ shunt reoperation, fontanelle bulging due to persistent hydrocephalus progressed to macrocephaly with bony defect and may have played a critical role in the development of craniocerebral disproportion.

Piatt coined the term "craniocerebral disproportion" to indicate situations where there is a gross discrepancy between the volume of the brain and the volume of the cranium [1]. The consequences of this craniocerebral disproportion include mechanical instability of the cortical mantle that may result in non-fused fontanelle with bony defect and macrocephaly.

As in the current case, when long-standing hydrocephalus is resolved by using CSF diversion treatment, reduction cranioplasty can be the optimal treatment option for correcting macrocephaly that accompanies bony defect $[1,2,5,10,11]$. In our case, we used the pi-technique to perform reduction cranioplasty, thereby correcting the hydrocephalic macrocephaly and non-fused fontanelle with bony defect. The result was good, with satisfactory bony defect closure and skull vault reshaping.

The pi-procedure consists of initial craniectomies designed in the shape of the Greek letter " $\pi$ " (pi), followed by extensive bone-to-dura undermining. Gentle twisting of wires in the region of the Bregma is done, and the antero-posterior (AP) diameter is shortened [12-14]. The original technique was modified to advance the bilateral coronal-parietal flap to the midline, rather than removing it, in order to cover the wide bone defect at the anterior fontanelle with the additional bone harvested from the paired sagittal ostectomy.

Many potential risks associated with cranial reduction surgery have been reported in the literature, including significant blood loss, air embolism, venous infarction, infection, neurological deficit, and death [10, 13]. However, we have experienced no specific complications after reduction cranioplasty utilizing the pi-technique. 
To summarize the case in the context of managing an infant with Chiari malformation type I, removal of the occipital encephalocele was undertaken initially to treat the various congenital anomalies. Next, various CSF diversion procedures such as shunt operation, foramen magnum decompression, and ETV were performed to treat hydrocephalus. However, shunt malfunction and infection developed and resulted in long-standing hydrocephalus, and although the long-standing hydrocephalus improved, macrocephaly with non-fusing fontanelle and subsequent bony defect developed.

In such circumstances, we recommend reduction cranioplasty utilizing the modified pi-technique to achieve a satisfactory outcome.

\section{References}

1. Piatt JH Jr, Arguelles JH (1990) Reduction cranioplasty for craniocerebral disproportion in infancy: indications and technique. Pediatr Neurosurg 16:265-270

2. Ehni G (1982) Reduction of head size in advanced hydrocephalus: a case report. Neurosurgery 11:223-228

3. Sabry MZ, Wornom IL 3rd, Ward JD (2001) Results of cranial vault reshaping. Ann Plast Surg 47:119-125, discussion 126
4. Gutierrez FA, McLone DG, Raimondi AJ (1979) Physiopathology and a new treatment of chronic subdural hematoma in children. Childs Brain 5:216-232

5. Matsui T, Tsutsumi K, Kaizu H, Asano T (2001) Reduction cranioplasty for craniocerebral disproportion due to chronic subdural hematoma in infants. A technical report. Neurol Res 23:67-71

6. Castillo M, Quencer RM, Dominguez R (1992) Chiari III malformation: imaging features. Am J Neuroradiol 13:107-113

7. Agrawal A, Mittal A, Kohali GB, Sampley S, Gupta A (2011) Chiari III malformation. Pediatr Neurosurg 47:309-310

8. Rani H, Kulkarni AV, Rao RV, Patil P (2013) Chiari III Malformation: a rare case with review of literature. Fetal Pediatr Pathol 32:169-174

9. Di Rocco C, Frassanito P, Massimi L, Peraio S (2011) Hydrocephalus and Chiari type I malformation. Childs Nerv Syst ChNS Off J Int Soc Pediatr Neurosurg 27:1653-1664

10. Park TS, Grady MS, Persing JA, Delashaw JB (1985) One-stage reduction cranioplasty for macrocephaly associated with advanced hydrocephalus. Neurosurgery 17:506-509

11. Sundine MJ, Wirth GA, Brenner KA, Loudon WG, Muhonen MG, Greene CS, Rowen S, Sabzghagaei N (2006) Cranial vault reduction cranioplasty in children with hydrocephalic macrocephaly. J Craniofac Surg 17:645-655

12. Wexler A, Cahan L (2012) The Venetian blind technique: modification of the pi procedure for the surgical correction of sagittal synostosis. J Craniofac Surg 23:2047-2048

13. Georgiev K, Enchev Y, Bussarsky A (2009) Operative technique for calvarial reconstruction of sagittal craniosynostosis. Turk Neurosurg 19:58-62

14. Guimaraes-Ferreira J, Gewalli F, David L, Olsson R, Friede H, Lauritzen CG (2001) Clinical outcome of the modified pi-plasty procedure for sagittal synostosis. J Craniofac Surg 12:218-224, discussion 225-216 